\title{
Commentary \\ Resuscitation of hemorrhagic shock with normal saline versus lactated Ringer's: effects on oxygenation, extravascular lung water, and hemodynamics
}

\author{
Samir G Sakka
}

Department of Anesthesiology and Intensive Care Medicine, Medical Center Cologne-Merheim, University of Witten/Herdecke, Ostmerheimerstrasse 200, 51109 Cologne, Germany

Corresponding author: Samir G Sakka, SakkaS@Kliniken-Koeln.de

Published: 3 April 2009

Critical Care 2009, 13:128 (doi:10.1186/cc7742)

This article is online at http://ccforum.com/content/13/2/128

(c) 2009 BioMed Central Ltd

See related research by Phillips et al., http://ccforum.com/content/13/2/R30

\begin{abstract}
Which type of fluid to use in the resuscitation from hemorrhagic shock, within and between crystalloids or colloids, is still a matter of debate. In this context, with respect to organ dysfunction, early detection of lung injury is widely considered of particular clinical importance. For these purposes, the transpulmonary thermodilution technique that enables one to assess extravascular lung water as a marker of pulmonary edema is applied in the clinical setting. In this issue of Critical Care, Phillips and colleagues describe that early resuscitation of hemorrhagic shock in pigs with two different crystalloid solutions - normal saline or Ringer's lactate - had little impact on oxygenation when the resuscitation volume was $<250 \mathrm{ml} / \mathrm{kg}$. Ringer's lactate had more favorable effects than normal saline, however, on extravascular lung water, $\mathrm{pH}$, and blood pressure but not on oxygenation. Although several pathophysiological aspects remain unanswered, these data are interesting in so far as they indicate that clinically applied amounts of crystalloids per se do not negatively influence pulmonary function, while with larger amounts the type of fluid has different effects on the extent of fluid extravasation in the lungs.
\end{abstract}

In the present issue of Critical Care an experimental model of hemorrhagic shock in pigs describes a significant difference in the extent of lung injury as assessed by the extravascular lung water (EVLW) between two crystalloid solutions normal saline (NS) or Ringer's lactate (RL) - when administered in volumes $>250 \mathrm{ml} / \mathrm{kg}$ [1]. In general, which type of fluid to use in the resuscitation from hemorrhagic shock is still a matter of debate. In this context, with respect to developing organ dysfunction, early detection of lung injury during resuscitation from hemorrhagic shock is widely considered of particular clinical importance. For these purposes, the transpulmonary thermodilution technique which enables one to assess the extent of fluid in the interstitial space of the lungs (EVLW) by describing the relation between intravascular fluid and extravascular fluid (that is, pulmonary capillary permeability) - is clinically applied. Especially in the scenario of fluid shift during resuscitation in hemorrhagic shock, the type of fluid may have particular influence on endothelial function in the lungs.

The study of Phillips and colleagues attempted to mimic severe hemorrhagic shock in both the prehospital and earlyhospital periods in humans [1]. Studies examining the effects of NS versus RL on hemodynamic response have so far been conducted in controlled hemorrhage models, and many studies reinfused shed blood with the resuscitation fluid. In contrast, this study used a more clinically relevant model by adding tissue injury to uncontrolled hemorrhage and initiating early resuscitation with crystalloids alone and resuscitating to a goal blood pressure. As the authors themselves mention, however, their study has several limitations that make the interpretation of the effects of the fluid type independent of the volume difficult. Nevertheless, the results probably allow more reliable extrapolation to the human clinical scenario than previous studies.

First, total blood loss was greater in the NS group than in the $\mathrm{RL}$ group, which may impact on the study findings. Furthermore, although animals in the NS received significantly more fluid, the mean arterial pressure was significantly lower in the resuscitation phase when compared with the $\mathrm{RL}$ group. At a time point during resuscitation when differences between both groups in EVLW first became significant, however, a higher capillary permeability as assessed by the index parameter pulmonary capillary permeability was already observed. Significantly higher pulmonary capillary permeability in the presence of a comparable filling volume implies either greater 
extravasation of fluid into the lung due to increases in permeability, or changes in the transcapillary oncotic pressure gradient, or an impairment of fluid clearance, or a combination of the three.

In general, the various factors for transcapillary fluid flux have been described in the Starling equation. Since this occurred at a time when there were no differences in the volumes of resuscitation administered or in the central filling volumes, however, this finding suggests that NS may have caused a pulmonary capillary endothelial permeability injury relative to the RL group to explain the differences in EVLW. It should be mentioned that nobody has previously examined EVLW, oxygenation, and hemodynamic effects in an uncontrolled trauma-related hemorrhagic shock model.

With respect to the monitoring technology used, a clinical study compared NS with different colloids and found that pulmonary edema (that is, EVLW as measured by transpulmonary thermodilution) and the lung injury score were not affected by the type of fluid loading in fluid responsiveness in both septic patients and nonseptic patients [2]. van der Heijden and colleagues, however, did not compare different crystalloids and did not involve individuals with a different underlying pathophysiology [2]. Phillips and colleagues present evidence that the difference in EVLW between NS versus $\mathrm{RL}$ may have been due to increased pulmonary vascular permeability [1] - surprisingly, there is little in the literature describing this effect. Further pathophysiological mechanisms to explain the authors' findings are peripheral vasodilatation by NS and acidosis, which itself may cause vasodilation and impair endothelial integrity [3].

Interestingly, oxygenation $\left(\mathrm{PaO}_{2}\right.$ to $\mathrm{FiO}_{2}$ ratio) was not significantly different between both groups while EVLW was. Previous clinical and experimental studies showed that transpulmonary thermodilution-derived EVLW is sufficiently accurate when compared with gravimetry or with the doubleindicator dilution technique [4-6]. Consequently, to explain this difference and to exclude limitations by the technique itself, a reference technique - such as gravimetry, which would have been easy to apply in an animal study - would have been useful. The authors adequately mentioned in their discussion the following possible explanations for the differences observed between NS and RL: inflammation, oncotic pressure (which unfortunately was not measured) and different influences on coagulation and platelet activation. Histological examinations that were obtained by the same group in the same model [7], however, revealed that increased numbers of sequestered neutrophils in the lung between NS-resuscitated and RL-resuscitated animals were not different. Finally, ventilator settings that may have influenced the study results were discussed. Although clinically not recommended and hopefully not applied, an identical tidal volume of $12 \mathrm{ml} / \mathrm{kg}$ was used in both groups. According to the authors, this tidal volume may have caused some lung injury and may have played a role in the development of increased EVLWI; however, using the same tidal volume made it unlikely as the cause of the difference between the groups.

Further, more sophisticated studies are required to validate and explain these findings. The authors are to be congratulated for completing this first phase of a promising line of investigation. Future research should further clarify the clinical significance of different fluids on pulmonary capillary function in patients with hemorrhagic shock.

\section{Competing interests}

SGS is a member of the medical advisory board of Pulsion Medical Systems AG, Munich, Germany, and received honoraria for presenting lectures.

\section{References}

1. Phillips CR, Vinecore K, Hagg DS, Sawai RS, Differding JA, Watters JM, Schreiber MA: Resuscitation of hemorrhagic shock with normal saline vs. lactated Ringer's: effects on oxygenation, extravascular lung water, and hemodynamics. Crit Care 2009, 13:R30.

2. van der Heijden M, Verheij J, van Nieuw Amerongen GP, Groeneveld AB: Crystalloid or colloid fluid loading and pulmonary permeability, edema, and injury in septic and nonseptic critically ill patients with hypovolemia. Crit Care Med 2009. [Epub ahead of print]

3. Maniatis NA, Kotanidou A, Catravas JD, Orfanos SE: Endothelial pathomechanisms in acute lung injury. Vascul Pharmacol 2008, 49:119-133

4. Katzenelson R, Perel A, Berkenstadt H, Preisman S, Kogan S, Sternik L, Segal E: Accuracy of transpulmonary thermodilution versus gravimetric measurement of extravascular lung water. Crit Care Med 2004, 32:1550-1554.

5. Sakka SG, Rühl CC, Pfeiffer UJ, Beale R, McLuckie A, Reinhart K, Meier-Hellmann A: Assessment of cardiac preload and extravascular lung water by single transpulmonary thermodilution. Intensive Care Med 2000, 26:180-187.

6. Neumann P: Extravascular lung water and intrathoracic blood volume: double versus single indicator dilution technique. Intensive Care Med 1999, 25:216-219.

7. Watters JM, Brundage SI, Todd SR, Zautke NA, Stefater JA, Lam JC, Muller PJ, Malinoski D, Schreiber MA: Resuscitation with lactated ringer's does not increase inflammatory response in a Swine model of uncontrolled hemorrhagic shock. Shock 2004, 22:283-287. 